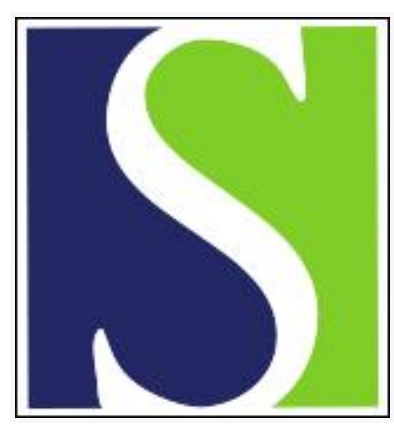

Scand J Work Environ Health 1999;25(6):564-568

https://doi.org/10.5271/sjweh.481

Issue date: Dec 1999

New avenues in research on musculoskeletal disorders

by Viikari-Juntura E, Riihimäki H

The following articles refer to this text: 2003;29(2):124-133;

2016;42(2):103-124

Key terms: assessment of outcome; exposure assessment; genetic epidemiology; low back; psychological factor; work organizational factor

This article in PubMed: www.ncbi.nlm.nih.gov/pubmed/10884154

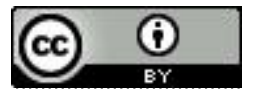




\title{
New avenues in research on musculoskeletal disorders
}

\author{
by Eira Viikari-Juntura, DMedSci, ${ }^{\dagger}$ Hilkka Riihimäki, DMedSci'
}

\begin{abstract}
Viikari-Juntura E, Riihimäki H. New avenues in research on musculoskeletal disorders. Scand $J$ Work Environ Health 1999;25(6, special issue):564-568.

Recent reviews on musculoskeletal disorders have clearly pointed out the need for more good-quality original research. Both symptom-based and objective outcome assessment methods should be improved. A variety of methods exists to assess exposure for short-term outcomes, whereas exposure assessment strategies for outcomes with long induction times should be developed. The change in exposure patterns - less force demands, more static, visual, cognitive, psychological and social load - will continue during the next millennium, and a likely result is a change in the disease panorama. The pathomechanisms of the effects of low-level static contractions and adverse psychosocial conditions need clarification. Genetic epidemiology is an emerging field of research, and it will be particularly interesting to study the interaction between genetically determined susceptibility and occupational risk factors. Because degeneration of the spine starts in adolescence, the importance of studying the etiology of degenerative disorders among young populations is emphasized.
\end{abstract}

Key terms assessment of outcome, exposure assessment, genetic epidemiology, low back, psychological factors, work organizational factors.

The number of articles on musculoskeletal disorders has grown remarkably during recent years, maybe more than in many other fields of occupational medicine. Towards the end of this millennium, published articles have in creasingly been reviews. The reviews on epidemiologic studies on musculoskeletal disorders differ in their conclusions about the work-relatedness of various disorders, especially as regards the causal role of work-related risk factors. Common to systematic critical reviews is a low number of original papers with high quality scores. Therefore our knowledge on risk factors is largely based on studies with moderate-to-low quality. Against this background the research needs are clear: more goodquality original research. This need creates demands on the assessment of the outcomes and exposures, study designs, and conceptual models.

\section{Assessment of outcome}

We feel that the development of the methods to assess the outcomes for research in the musculoskeletal area should be much more in focus. No matter how simple or sophisticated the assessment method (questionnaire, interview, physical examination, imaging, electrodiag- nostic assessment), we should use validated methods that can correctly classify states of disease with different severity and prognosis. Due to the deficient knowledge of how to classify musculoskeletal disorders, unspecified symptoms have generally been used as indicators. For example, as regards the low back and the neck, it has been feasible to differentiate between radiating and nonradiating pain. These 2 types have a different prognosis, and there is some indication that they may be etiologically different (1). No generally accepted classification system exists, however. Research applying qualitative methods could provide a new approach to learning more about musculoskeletal disorders and provide a guide to better classification systems. Basic science is needed to elucidate structural changes in tissues in association with disease.

Most of the currently used physical examination methods were described decades ago for the most common disorders, and the methods have been in routine clinical use for decades. Still today their performance has not been standardized in enough detail. Interexaminer repeatability is also either insufficient or unknown for many of these tests $(2,3)$. The possibilities that modern imaging methods allow have been little used in the research of musculoskeletal disorders. With developing technology, their use will be more feasible in the future.

1 Finnish Institute of Occupational Health, Helsinki, Finland.

Reprint requests to: Dr Eira Viikari-Juntura, Musculoskeletal Research Unit, Department of Physiology, Finnish Institute of Occupational Health, Topeliuksenkatu 41 a A, FIN-00250 Helsinki, Finland. [E-mail: eira.viikari-juntura@occuphealth.fi] 
Still, we do not yet understand well what we see, for instance, in the magnetic resonance image (MRI). Validation of MRI findings against pathoanatomical and histological findings would be a natural first step - on a long and burdensome road.

Nerve conduction studies have been mostly used for the case definition of nerve entrapment syndromes, especially carpal tunnel syndrome (CTS). According to a recent consensus paper, the diagnosis of CTS in epidemiologic studies should be based on symptoms and electrodiagnostic findings (4). Yet the criteria for a case differ markedly between studies, and the advantages and drawbacks of various criteria have not been thoroughly discussed. For example, the appropriateness of a difference in nerve conduction between the median and ulnar nerve as the sole basis of CTS diagnosis in epidemiologic studies can be questioned on the basis of the results of Stetson et al (5). These authors studied industrial workers and managerial or professional employees and found that nerve conduction measures differed between the 2 groups not only for the median but also for the ulnar nerve. The performance of nerve conduction studies is also a technically demanding procedure. One can say that a low-cost solution to improve the knowledge of the etiology of CTS or other nerve entrapments is unlikely to exist.

In the neck and low-back area, the analysis of the electromyographic spectrum has given much insight into local muscular fatigue, and it can be used as an objective indicator of fatigue. In the neck area, most studies have collected information from one particular muscle, the trapezius, and much less is known about the more deeply lying muscles. With needle electromyography, knowledge has been collected about the events in deep muscles and for single motor units. Such studies have shown that, at the motor unit level, a recruitment pattern exists, some motor units being recruited first and unrecruited last, leading to fatigue at the single motor unit level (6). Simultaneous recordings of several muscles around the shoulder during fatiguing contractions have shown no constant order in the development of fatigue between the muscles or muscle parts, and it is possible that, to avoid fatigue, the load is transferred from one muscle to another during contraction (7). This assumption suggests that the development of fatigue may be a complex phenomenon, and surface electromyography of one or a few muscles may be a poor reflector of loading and fatigue for large studies.

\section{Exposure assessment}

The assessment of physical loads has been under much more extensive development during the end of this millennium than the assessment of musculoskeletal outcomes has. Numerous validity studies have enabled us to see how detailed information can be obtained by questionnaires (8), interviews (9), observations (10), and direct measurements (11). More importantly, the strategy of exposure assessment has been brought into focus, and it has been emphasized that we should be realistic with our endeavors in exposure assessment in epidemiologic studies (12). Our ability to assess cumulative work load is still in its infancy, and methods should be developed and used for outcomes with long induction times. Job exposure matrices may be one solution for exposure assessment in large epidemiologic studies. So far, such data on physical load factors have been crude. With accumulating data from surveys and other studies we expect the quality of these data to improve after the turn of the millennium.

\section{Will changes in exposure patterns cause a change in the disease panorama?}

The changes in exposure in many jobs during recent years - less force demands and more static, visual, cognitive, psychological and social load - will undoubtedly continue. In fact it is difficult to foresee any point in the near future when this development would cease.

At the same time the human being has undergone only small changes. In developed countries the nutrition of the population has improved, resulting in an increase in average height, an increase in average age, and a decrease in or disappearance of some serious disorders, such as tuberculosis and rickets. Unfortunately, these positive trends have been accompanied by some negative ones, such as increasing overweight and a sedentary life-style.

These changes in exposure and in the human being are likely to affect the disease panorama. We have so far seen that the prevalence of self-reported back pain has been rather stable over a 14-year period from 1979 to 1992 in the Finnish general population (13). The prevalence of continuous ache was stable also in an interview survey by Statistics Finland among wage earners during a 20-year period from 1977 to 1997 in all other body regions but the neck, in which a clear increase was seen among both men and women (14). It remains to be shown whether there have been changes in the grade of osteoarthrosis or disc degeneration of populations with time. One may say - unfortunately not with good supporting data - that there has been and will probably be a change in the panorama also of upper-limb disorders. Epidemics of tenosynovitis or peritendinitis, such as were seen in the postwar auto industry (15), have not been reported lately, in the era of the "mouse hand" - whatever the concept may entail. Surveillance systems sensitive for 
changes should be used to obtain information about the development in both exposure and disease panorama.

\section{Role of work organizational and psychological factors}

The role of work organizational and psychological factors has been a topic of interest in musculoskeletal research for some decades. For work organizational factors, the demand-control-support model (16) that has been extensively used in research on cardiovascular disorders has been mostly applied. Bongers and her coworkers (17) presented a review on epidemiologic studies investigating the associations between psychosocial factors and musculoskeletal disorders. They found some evidence for associations between musculoskeletal disorders and work demands, control, and social support. Individual characteristics such as psychological and emotional problems have shown more consistent associations with more evidence also from longitudinal studies. Stress symptoms have preceded musculoskeletal symptoms in several longitudinal studies, and, in the conceptual model presented by the authors, stress symptoms were considered to be a mediator of the effects of work organizational factors. A major future research question is the extent to which work organizational and psychological factors are causal with respect to musculoskeletal disorders and modifiers of illness behavior. Although it is certainly possible to study the effects of these factors with more rigorous study designs and well-defined outcomes, the dynamic process of the events with different coping and feedback mechanisms calls also for other research paradigms, different from those of traditional epidemiology. New knowledge is needed to learn about the potential of primary and secondary prevention.

The changes in the disease panorama and the multitude of recognized risk factors create research needs in the pathomechanical processes: what are the effects of low-level static loads, visual strain, and psychological strain in the musculoskeletal organs? How are the endocrinological functions associated with these phenomena?

There is a vast body of literature on the effects of mental demands and work organizational factors on neuromuscular function. There is evidence that motivational factors and mental states (anger, hostility, and fear) can increase muscle activation (18). A motor system of predominantly monoaminergic descending pathways from the brain stem has been suggested to initiate or control motor responses of emotional stimuli. At the peripheral level, in association with low contraction levels, the hypothesis that the recruitment order in a contracting muscle is constant with small units recruited first and staying active the longest, and that this phenomenon is associated with neck disorders, has gained support even in a longitudinal study (19). Several other hypotheses exist on pain mechanisms (20) which should be tested in the future. The metabolic effects of adverse psychosocial conditions have been explained by catabolism and have, in some studies, been seen as a decreased production of anabolic hormones, such as testosterone (21). However, the associations between musculoskeletal pain and the levels of such hormones have not been consistent in studies and should be investigated more rigorously.

\section{Genetic epidemiology in musculoskeletal disorders}

Recent fast progress in molecular genetic techniques has resulted in the ambitious Human Genome Project that will map and sequence the human genome. This project is expected to be completed during the first decade of the new millennium. It opens a new avenue of genetic epidemiology also for researchers of musculoskeletal disorders. There is evidence that degenerative disorders of the musculoskeletal system, such as disc degeneration and osteoarthrosis, are influenced by hereditary factors $(22,23)$. A few studies have already appeared showing that mutations of the vitamin $\mathrm{D}$ receptor gene are associated with disc degeneration (24) and osteoarthritis (25), and mutations of the type IX collagen gene are associated with degeneration of articular cartilage and intervertebral discs (26). It is likely that also other musculoskeletal disorders are influenced by genetic factors.

It will be particularly interesting to study the interaction between genetically determined susceptibility and occupational risk factors. Case-control studies are the most suitable to investigate genetic risk factors and their interactions with other risk factors of a disease (27). In case-control studies several candidate genes can be tested. Such testing is made easy by modern molecular genetic techniques, such as DNA (deoxyribonucleic acid) chips which allow the identification of several mutations of genes at low cost.

\section{Role of major and minor traumas}

The assessment of the role of various types of major and minor traumas in back and limb disorders has been one of the most difficult areas in research on musculoskeletal disorders. As accidents or losses of balance are rare stochastically occurring events, it is difficult to record and characterize them systematically for epidemiologic purposes. Although we believe in a major role of trauma 
in the etiology of musculoskeletal disorders, convincing scientific proof on how much importance various types of traumas have for different outcomes is lacking. This question should be rigorously tackled with prospective studies. Future development in modern technology may make it possible to detect minor injuries in the intervertebral disc or cartilage or other tissues.

\section{Workplace modifications central to primary and secondary prevention}

We believe that primary prevention will always be more effective than secondary prevention. The high occurrence of musculoskeletal disorders shows that primary prevention has so far not been very successful. A third, and among some worker groups almost half, of all days of sick leave are due to musculoskeletal disorders.

As primary care physicians see patients with musculoskeletal disorders almost daily, some of them with a recent onset of symptoms, they have a unique possibility to initiate secondary preventive measures often at a relatively early stage of the disease. As we have convincing evidence that physical work load factors, if not causative, can trigger or aggravate most musculoskeletal disorders, directing secondary preventive measures towards workplace adjustment should be a first priority for treatment, and in some cases a major component of treatment. This practice has not been common so far, but it should be so in the future. The process of making changes at the workplace is not simple and requires co-operation of the employer and often several bodies at the workplace. Investigating the effects of intervention requires a multidisciplinary approach and the application of different research paradigms.

\section{New knowledge from young populations}

Most epidemiologic studies on the etiology of low-back disorders have been carried out in working age populations. A large number of risk factors, both work- and person-related, have been identified. Accordingly, preventive programs and efforts have mainly been targeted toward the working-age population. During the past 10 years evidence has accumulated that disc degeneration of the lumbar spine and low-back pain start to appear already in adolescence $(28,29)$. This occurrence raises the question of whether the preventive programs should actually be directed toward young people. For this end, we should learn more about the risk factors of low-back disorders among the young. Probably the best way to proceed would be to start prospective cohort studies with repeated assessments of risk factors and disease outcomes.

\section{References}

1. Luoma K, Riihimäki $H$, Luukkonen R, Raininko R, ViikariJuntura $E$, Lamminen A. Low back pain in relation to lumbar disc degeneration. Spine. In press.

2. Marx RG, Bombardier C, Wright JG. What do we know about the reliability and validity of physical examination tests used to examine the upper extremity? J Hand Surg 1999;24A:18593.

3. Deyo R, Rainville J, Kent D. What can the history and physical examination tell us about low back pain? JAMA 1992; 268:760-5.

4. Rempel D, Evanoff B, Amadio PC, de Krom M, Franklin G, Franzblau A, et al. Consensus criteria for the classification of carpal tunnel syndrome in epidemiologic studies. Am J Public Health 1998;88:1447-51.

5. Stetson DS, Silverstein BA, Keyserling WM, Wolfe RA, Albers JW. Median sensory distal amplitude and latency: comparisons between nonexposed managerial/professional employees and industrial workers. Am J Ind Med 1993;24:17589.

6. Henneman 1965, Somjen G, Carpenter DO. Excitability and inhibitability of motoneurons of different sizes. J Neurophysiol 1965;28:599-620.

7. Nieminen H, Takala E-P, Niemi J, Viikari-Juntura E. Muscular synergy in the shoulder during a fatiguing static contraction. Clin Biomech 1995;10:309-17.

8. Hollman S, Klimmer F, Schmidt K-H, Kylian H. Validation of a questionnaire for assessing physical work load. Scand J Work Environ Health 1999;25(2):105-14.

9. Wiktorin C, Selin K, Ekenvall L, Alfredsson L, Music-Norrtälje Group. An interview technique for recording work postures in epidemiological studies. Int J Epidemiol 1996; 25:171-80.

10. Kilbom $\AA$. Assessment of physical exposure in relation to work-related musculoskeletal disorders - what information can be obtained from systematic observations? Scand J Work Environ Health 1994;20 special issue:30-45.

11. Fathallah FA, Marras WS, Parnianpour M. An assessment of complex spinal loads during dynamic lifting tasks. Spine 1998;23:706-16.

12. Burdorf $A$. Reducing random measurement error in assessing postural load on the back in epidemiologic surveys. Scand J Work Environ Health 1995;21:15-23.

13. Leino P, Berg M-A, Puska P. Is back pain increasing? Results from national surveys in Finland during 1978/9-1992. Scand J Rheumatol 1994;23:269-76

14. Lehto A-M, Sutela H. Efficient, more efficient, exhausted: finding of Finnish quality of work life surveys 1977-1997. Helsinki: Labour Market, Statistics Finland, 1999:8.

15. Thompson AR, Plewes LW, Shaw EG. Peritendinitis crepitans and simple tenosynovitis: a clinical study of 544 cases in industry. Br J Ind Med 1951;8:150—8.

16. Karasek RA, Theorell T. Healthy work. New York (NY): Basic Books, 1990.

17. Bongers PM, de Winter CR, Kompier MAJ, Hilderbrandt VH. Psychosocial factors at work and musculoskeletal disease [review]. Scand J Work Environ Health 1993;19:297312 . 
18. Westgaard RH. Effects of psychological demand and stress on neuromuscular function. In: Moon SD, Sauter SL, editors. Psychosocial aspects of musculoskeletal disorders in office work. London: Taylor et Francis 1996:75-89.

19. Veiersted KB, Westgaard RH, Andersen P. Electromyographic evaluation of muscular work pattern as a predictor of trapezius myalgia. Scand J Work Environ Health 1993;19:28490.

20. Johansson H, Sojka P. Pathophysiological mechanisms involved in genesis and spread of muscular tension in occupational muscle pain and in chronic musculoskeletal pain syndormes: a hypothesis. Med Hypotheses 1991;35:196-203.

21. Theorell T, Karasek RA, Eneroth P. Job strain variations in relation to plasma testosterone fluctuations in working men: a longitudinal study. J Intern Med 1990;227:31-6.

22. Felson DT. Epidemiology of hip and knee osteoarthritis. Epidemiol Rev 1988;10:1-28.

23. Battié MC, Kaprio J. The role of genetic influences in disk degeneration and herniation. In: Weinstein $\mathrm{JN}$, Gordon $\mathrm{S}$, editors. Low back pain: a scientific and clinical overview. Rosemont (IL): American Association of Orthopaedic Surgeons 1996:85-96.
24. Videman T, Leppävuori J, Kaprio J, Battié MC, Gibbons LE, Peltonen L, et al. Intragenic polymorphisms of the vitamin D receptor gene associated with intervertebral disc degeneration. Spine 1998;23:2477-85.

25. Keen RW, Hart DJ, Lanchburry JS, Spector TD. Association of early osteoarthritis of the knee with a Taq I polymorphism of the vitamin $D$ receptor gene. Arthritis Rheum 1997;40:1444-9.

26. Kimura T, Nakata K, Tsumaki N, Miyamoto S, Matsui Y, Ebara $\mathrm{S}$, et al. Progressive degeneration of articular cartilage and intervertebral discs: an experimental study in transgenic mice bearing a type IX collagen mutation. Int Orthop 1996;20:177-81.

27. Khoury MJ. Genetic epidemiology. In: Rothman K, Greenland S, editors. Modern epidemiology. Philadelphia (PA) Lippincott-Raven, 1998:609-21.

28. Duggleby T, Kumar S. Epidemiology of juvenile low back pain: a review. Disability Rehabil 1997;19:502-12.

29. Salminen JJ, Erkintalo M, Laine M, Pentti J. Low back pain in the young. A prospective three-year follow-up study of subjects with and without low back pain. Spine 1995;20:21017. 